\title{
Impact of a surgical intensivist on the clinical outcomes of patients admitted to a surgical intensive care unit
}

\author{
Chi-Min Park ${ }^{1,2}$, Ho-Kyung Chun ${ }^{3}$, Dae-Sang Lee ${ }^{1}, K_{y e o n g m a n} J^{1,4}$, Gee Young Suh ${ }^{1,4}$, Jin Cheol Jeong ${ }^{5}$ \\ Departments of ${ }^{1}$ Critical Care Medicine and ${ }^{2}$ Surgery, Samsung Medical Center, Sungkyunkwan University School of Medicine, \\ Seoul, ${ }^{3}$ Department of Surgery, Kangbuk Samsung Medical Center, Sungkyunkwan University School of Medicine, Seoul, ${ }^{4}$ Division \\ of Pulmonary and Critical Care Medicine, Department of Medicine, Samsung Medical Center, Sungkyunkwan University School of \\ Medicine, Seoul, ${ }^{5}$ Department of Surgery, Hallym University Kangdong Sacred Heart Hospital, Hallym University School of \\ Medicine, Seoul, Korea
}

\begin{abstract}
Purpose: An intensivist is a key factor in the mortality of patients admitted to the intensive care unit (ICU). The aim of this study was to evaluate the effect of an intensivist on clinical outcomes of patients admitted to a surgical ICU.

Methods: During the study period, the surgical ICU was converted from an open ICU to an intensivist-directed ICU managed by an intensivist who was board certified in both general surgery and critical care medicine. We compared consecutive patients admitted to the surgical ICU before and after implementing the intensivist-directed care. The primary outcome was ICU mortality, and secondary outcomes were hospital mortality, 90-day mortality, length of hospital stay, ICU-free days, ventilator-free days, and ICU readmission rate.

Results: A total of 441 patients were included in this study: 188 before implementation of the intensivist and 253 after implementation. Clinical characteristics were not different between the two groups. ICU mortality decreased from $11.7 \%$ to $6.3 \%(P=0.047)$ after implementation, and 90-day mortality also decreased significantly $(P=0.008)$. The adjusted hazard ratio of the intensivist for ICU mortality was 0.43 (95\% confidence interval, $0.22-0.87 ; P=0.020)$. ICU-free days $(P=0.013)$ and the hospital length of stay ( $\mathrm{P}=0.032)$ were significantly improved after implementing the intensivist-directed care. Before implementation period, $16.0 \%$ of patients were readmitted, compared with only $9.9 \%$ after implementation $(P=0.05)$. Conclusion: Implementing intensivist-directed care in the surgical ICU was associated with significant improvements in ICU mortality and significant clinical outcomes.

[Ann Surg Treat Res 2014;86(6):319-324]
\end{abstract}

Key Words: Intensive care units, Critical illness, Specialization, General surgery, Mortality

\section{INTRODUCTION}

Intensivists are physicians who specialize in the care of critically ill patients and who direct and provide critical care in an intensive care unit (ICU) [1]. In the past, many ICUs used the open ICU model in which patients were cared for by their primary care physician. In the 2000s, as the positive impact of a qualified intensivist on the outcome of ICU patients became recognized [2], many hospitals adopted a high-intensity or closed ICU model in which patients are directed exclusively by an intensivist [1,3]. For surgical ICUs, there are several published reports suggesting improved outcomes in patients managed by an intensivist [4-9].

Despite these positive impacts, high-intensity ICUs managed by intensivists are not common in many hospitals, especially in surgical ICUs or in countries with limited medical resources
Received December 11, 2013, Revised January 29, 2014,

Accepted February 7, 2014

\section{Corresponding Author: Ho-Kyung Chun}

Department of Surgery, Kangbuk Samsung Medical Center,

Sungkyunkwan University School of Medicine, 29 Saemunan-ro, Jongno-

gu, Seoul 110-746, Korea

Tel: +82-2-2001-5100, Fax: +82-2-3410-0040

E-mail: hkchun@skku.edu
This study results were orally presented at the Annual Congress of Korean Surgical Society in November 2011

Copyright (c) 2014, the Korean Surgical Society

(c) Annals of Surgical Treatment and Research is an Open Access Journal. All articles are distributed under the terms of the Creative Commons Attribution NonCommercial License (http://creativecommons.org/licenses/by-nc/3.0/) which permits unrestricted non-commercial use, distribution, and reproduction in any medium, provided the original work is properly cited. 
$[10,11]$. Moreover, a recent large-scale study reported that hospital mortality was higher for patients managed by an intensivist than for those who were not [12]. The author speculated that this might reflect a disruption in management and a greater likelihood of miscommunication between the intensivist and primary physician [12].

In this study, by comparing the periods before and after a surgical ICU came under the direction of a surgical intensivist certified in both general surgery and critical care medicine, we evaluated the association between an intensivist-directed surgical ICU and clinical outcomes of admitted patients.

\section{METHODS}

\section{Study design}

This study was a retrospective cohort study designed to evaluate the effect of intensivist-directed care on ICU mortality and clinical outcomes in a surgical ICU at a cancer center of a university teaching hospital. The Institutional Review Board at Samsung Medical Center approved the study protocol.

Samsung Medical Center is a Sungkyunkwan University School of Medicine teaching hospital with 1,951 beds, located in Seoul, Korea. The Samsung Conprehensive Cancer Center is part of the Samsung Medical Center and was newly established in January 2008. The surgical ICU in this cancer center is a 13bed unit. Most patients are surgical patients that are admitted to the ICU following scheduled or unscheduled surgery. Postoperative patients that present with surgical complications or other medical problems are also admitted from the general wards or emergency room. Although this surgical ICU is located in the cancer center, a small number of non-oncology patients are also admitted.

When the cancer center was opened, the surgical ICU used an open ICU model. There was no critical care team, and the primary admitting surgeon and residents directly managed the patients with specialist consultation. In April 2009, the hospital decided to adopt the intensivist-directed ICU model, and the surgical ICU was gradually converted to an intensivistdirected ICU with completion in 2010, after which patients admitted to the surgical ICU were exclusively cared for by the surgical intensivist. This surgical intensivist was a surgeon who was board certified in both general surgery and critical care medicine. The surgical intensivist stayed in the ICU for more than 10 hours per day during every weekday and had no other clinical duties and took call overnight from home, returning to the ICU at their discretion. Daily rounds were performed by the surgical intensivist and the ICU team, which consisted of senior surgical residents and critical care nurses. Nurses were assigned to patients on a $1: 2$ or $1: 3$ ratio and worked 8-hour shifts; this nursing system was the same before and after conversion. After conversion to an intensivist-directed ICU, many practical protocols were implemented, such as sepsis management, lung protective ventilation, weaning protocol, sedation protocol, massive transfusion, and enteral and parenteral nutrition protocols. All were evidence-based protocols based on guidelines used worldwide. Although admission, discharge, procedure, and orders were mainly decided by the surgical intensivist and the ICU team, the intensivist usually communicated and discussed the treatment plan of the patients with primary surgeons at least once a day. Except for these changes implemented by the intensivist, there were no fundamental changes in the surgical ICU during the study duration.

\section{Study populations}

We compared patients admitted during two distinct phases: between January 2008 and December 2008, before implementing an intensivist-directed ICU model, and between January 2010 and December 2010, after complete conversion to an intensivist-directed ICU. We allowed a gradual transition period of one year to adopt the required changes.

Consecutive adult patients admitted to the surgical ICU during the two distinct phases were included in this study. The inclusion criteria were adult surgical patients older than 18 years who were admitted to the general surgical ICU for more than 48 hours during the study duration. We excluded patients who were admitted to the ICU for less than 48 hours because most of these patients were admitted not for intensive care, but for close monitoring of immediate postoperative complications. However, we included patients who died within 48 hours after ICU admission. Neurosurgery patients or thoracic surgery patients were not involved in this study because the thoracic and neurosurgical ICUs were separate from the surgical ICU. The total study population included 188 patients who were admitted before implementing an intensivist-directed ICU and 253 patients who were admitted after implementation.

\section{Data collection}

The following clinical data were retrospectively obtained from a prospectively collected ICU database and chart review: age, sex, main reason for ICU admission, hospital length of stay (LOS), ICU LOS, presence of cancer, comorbidity, duration of mechanical ventilation, and readmission to the surgical ICU during current hospital admission. Patients were followed until 90 days after ICU admission or death. To compare severity among patients admitted to the ICU, Acute Physiology and Chronic Health Evaluation (APACHE) II scores were calculated and compared.

\section{Statistical analysis}

The primary outcome was ICU mortality. The secondary outcomes were hospital and 90-day mortality, ICU-free days, ventilator-free days, and ICU re-admission. ICU mortality was 
defined as all deaths in the surgical ICU during the current hospitalization. Ventilator-free days were defined as the number of days between successful weaning from mechanical ventilation and day 28 after ICU admission [13]. ICU-free days were also defined as the number of days between ICU discharge and day 28 after ICU admission.

These variables were compared between the two phases before and after implementation of the intensivist-directed ICU (years 2008 and 2010). Categorical data were compared using a chi-square test or Fisher exact test. Continuous variables were compared with independent t-test or Mann-Whitney test, using two-sided testing. A P-value $<0.05$ was considered significant. The cumulative 90-day survival rate was obtained by the Kaplan-Meier method, and statistical significance was analyzed by the log-rank test. The effect of the intensivist on ICU, hospital, and 90-day mortalities was estimated using the Cox regression model with adjustment for the following clinically suspected variables: sex, comorbidity, presence of cancer, APACHE II score, unplanned admission, mechanical ventilation, and renal replacement therapy. Corresponding hazard ratio (HR) and 95\% confidence intervals (CIs) were reported. All analyses were conducted using PASW ver. 18.0 (SPSS Inc., Chicago, IL, USA).

\section{RESULTS}

\section{Clinical characteristics}

A total of 441 patients were enrolled in this study, 188 before implementing an intensivist-directed ICU and 253 after implementation. Baseline clinical characteristics are shown in Table 1.

The clinical characteristics including age, sex, and presence of oncologic disease were not different between the two periods. The cause of admission and location before admission were also not different. According to the operative data, operative time and estimated blood loss were not different between the two periods. The numbers of patients who received mechanical ventilation or continuous renal replacement therapy were not different between the periods. Mean APACHE II score was not statistically different between the two periods $(14.5 \pm 6.7$ vs. $15.2 \pm 7.8, \mathrm{P}=0.310$ ).

\section{Clinical outcomes}

ICU mortality, the primary outcome of this study, decreased from $11.7 \%$ to $6.3 \%$ ( $P=0.047)$ after implementing an intensivist-directed ICU (Table 2). The 90-day mortality decreased significantly from $18.6 \%$ to $10.3 \%(\mathrm{P}=0.012)$ (Fig.

Table 1. Demographic and clinical characteristics of patients admitted to the surgical ICU before and after implementing the intensivist-directed ICU

\begin{tabular}{|c|c|c|c|}
\hline Variable & $\begin{array}{c}\text { Before implementation ( } \mathrm{n} \\
=188 \text { ) }\end{array}$ & $\begin{array}{l}\text { After implementation } \\
\qquad(\mathrm{n}=253)\end{array}$ & P-value \\
\hline Age (yr) & $60.0 \pm 15.7$ & $61.5 \pm 13.8$ & 0.275 \\
\hline Sex (M:F) & $122: 66$ & $178: 75$ & 0.224 \\
\hline APACHE II & $14.5 \pm 6.7$ & $15.2 \pm 7.8$ & 0.310 \\
\hline \multicolumn{4}{|l|}{ Presence of cancer } \\
\hline Yes & 124 & 172 & 0.654 \\
\hline No & 64 & 81 & \\
\hline Cause of admission (\%) & & & 0.125 \\
\hline After scheduled surgery & $93(49.5)$ & $108(42.7)$ & \\
\hline After unscheduled surgery & $52(27.7)$ & $87(34.4)$ & \\
\hline Medical problem & $31(16.5)$ & $50(19.8)$ & \\
\hline Trauma without surgery & $12(6.4)$ & $8(3.2)$ & \\
\hline Location before admission & & & 0.077 \\
\hline Operation room & $143(76.1)$ & $191(74.6)$ & \\
\hline Emergency room & $21(11.2)$ & $21(8.3)$ & \\
\hline General ward & $22(11.7)$ & $37(14.6)$ & \\
\hline Other ICU & $1(0.5)$ & $4(1.6)$ & \\
\hline Other hospital & $1(0.5)$ & $0(0.0)$ & \\
\hline Mechanical ventilation & $69(36.7)$ & $93(36.8)$ & 0.990 \\
\hline Continuous renal replacement therapy & $11(5.9)$ & $15(5.9)$ & 0.973 \\
\hline Operation time (min) & $169(80-300)$ & $180(100-280)$ & 0.633 \\
\hline $\mathrm{EBL}(\mathrm{mL})$ & $400(100-700)$ & $300(75-600)$ & 0.277 \\
\hline ICU LOS (day) & $4(3-7)$ & $4(3-6)$ & 0.035 \\
\hline
\end{tabular}

Values are presented as mean \pm standard deviation, number (\%) or median (interquartile range).

APACHE, Acute Physiology and Chronic Health Evaluation; ICU, intensive care unit; EBL, estimated blood loss; LOS, length of stay. 
1), and hospital mortality also decreased from $14.9 \%$ to $9.9 \%$, although the change was not significant. The ICU-free days was significantly longer $(P=0.013)$ and the hospital LOS was significantly shorter $(\mathrm{P}=0.032)$ after implementing the intensivist-directed ICU. Thirty patients (16.0\%) were readmitted to the surgical ICU during the admission period before implementation of the intensivist-directed ICU, whereas only 25 patients $(9.9 \%)$ were readmitted after implementation ( $\mathrm{P}$ $=0.05)$. There was no significant difference in the number of ventilator-free days between the two groups $(P=0.203)$ (Table 2).

On Cox regression analysis, the unadjusted HR of intensivist for ICU mortality was $0.52(95 \% \mathrm{CI}, 0.27-1.04 ; \mathrm{P}=0.064)$ and adjusted HR was 0.43 ( $95 \% \mathrm{CI}, 0.22-0.87, \mathrm{P}=0.020$ ). The adjusted HR for 90-day mortality was $0.39(95 \% \mathrm{CI}, 0.23-0.67$, P $=0.001)$, and the adjusted HR for hospital mortality was 0.60 (95\% CI, 0.34-1.09, P = 0.095) (Table 3).

\section{DISCUSSION}

In this study, we compared the clinical outcomes between the periods before and after implementing an intensivistdirected ICU in a cancer center surgical ICU. The main finding of this study is that implementing an intensivist-directed

Table 2. Comparing the clinical outcomes of patients admitted to the surgical ICU before and after implementing an intensivist-directed ICU

\begin{tabular}{lccl}
\hline \multicolumn{1}{c}{ Variable } & $\begin{array}{c}\text { Before } \\
\text { implementation }\end{array}$ & $\begin{array}{c}\text { After } \\
\text { implementation }\end{array}$ & P-value \\
\hline Hospital LOS (day) & $33.8 \pm 29.9$ & $28.5 \pm 22.2$ & 0.032 \\
ICU-free day & $18.4 \pm 8.9$ & $20.4 \pm 7.5$ & 0.013 \\
Ventilator-free day & $23.8 \pm 8.1$ & $24.7 \pm 7.1$ & 0.203 \\
Readmission & $30(16.0)$ & $25(9.9)$ & 0.05 \\
Death & & & \\
ICU mortality & $22(11.7)$ & $16(6.3)$ & 0.047 \\
Hospital mortality & $28(14.9)$ & $25(9.9)$ & 0.109 \\
90-Day mortality & $35(18.6)$ & $26(10.3)$ & 0.012 \\
\hline
\end{tabular}

Values are presented as mean \pm standard deviation or number (\%).

LOS, length of stay; ICU, intensive care unit.
ICU was associated with significantly lower ICU mortality. There was also a significant decrease in 90-day mortality after implementation, and hospital mortality tended to decrease, although the change was not significant. Although there was no statistically significance in univariate analysis, multivariate analysis showed that the intensivist was independently related to ICU mortality. The study also showed that important clinical outcomes such as ICU-free days, hospital LOS, and ICU readmission were significantly improved after implementing the intensivist-directed ICU. These findings suggest that implementing an intensivist-directed ICU results in significantly improved clinical outcomes for patients admitted to a cancer center surgical ICU.

These findings are consistent with many studies that associate better outcomes with a intensivist and a higher level of intensity of ICU care [2]. According to the meta-analysis of Pronovost [2], hospital mortality was significantly decreased in $67 \%$ of studies, and the ICU mortality rate was significantly decreased in $75 \%$ of studies. The random-effects pooled estimates of the unadjusted relative risk of hospital and ICU mortality were $0.71(95 \% \mathrm{CI}, 0.62-0.82)$ and $0.61(95 \% \mathrm{CI}$, $0.50-0.75)$, respectively. The reason for the association between intensivist-directed ICU and improved clinical outcome is not fully understood. Possible explanations include the intensivists'

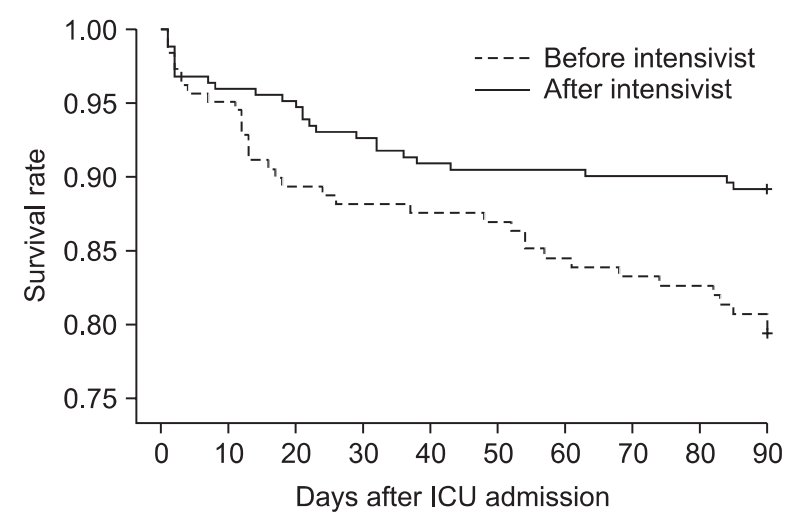

Fig. 1. Kaplan-Meier survival curve of the patients admitted to the surgical intensive care unit (ICU), before and after implementing an intensivist-directed ICU (Log-rank test, P = 0.013).

Table 3. Univariate and multivariate Cox regression analysis of intensivist with mortality

\begin{tabular}{lccccccc}
\hline & \multicolumn{3}{c}{ Univariate } & \multicolumn{3}{c}{ Multivariate } \\
\cline { 2 - 3 } & Unadjusted HR & $95 \% \mathrm{Cl}$ & P-value & Adjusted HR & $95 \% \mathrm{Cl}$ & P-value \\
\hline ICU mortality & 0.52 & $0.27-1.04$ & 0.064 & 0.43 & $0.22-0.87$ & 0.020 \\
Hospital mortality & 0.81 & $0.47-1.40$ & 0.456 & 0.60 & $0.34-1.09$ & 0.095 \\
90-Day mortality & 0.53 & $0.32-0.89$ & 0.015 & 0.39 & $0.23-0.67$ & 0.001 \\
\hline
\end{tabular}

$\mathrm{HR}$, hazard ratio; $\mathrm{Cl}$, confidence interval; $\mathrm{ICU}$, intensive care unit. 
availability and ability to prevent, recognize early, and treat life-threatening complications and to improve the compliance of evidence-based protocols [14,15]. Several reports have also shown that intensivists reduce the ICU LOS, thereby reducing the mortality rates associated with prolonged ICU stay [2]. In this study, the surgical intensivist performed daily rounds and stayed in the ICU during the daytime, and many evidencebased practical protocols were implemented and controlled by the intensivist. These factors may be associated with improved clinical outcomes of patients admitted in the intensivistdirected ICU.

In this study, implementing an intensivist-directed ICU model was associated with significantly shorter hospital LOS, more ICU-free days, and a lower rate of readmission. Decisions regarding ICU admission and discharge made by the intensivist may be related to decreasing ICU LOS and improving the overall ICU operation and bed turnover rate [16]. Although improvement of the bed turnover rate could increase readmission to the ICU because of early transfer to the general ward, in this study, both the ICU free-days and readmission rate were significantly improved after implementing an intensivistdirected ICU. This may have been due to adequate decisionmaking by the intensivist regarding the transfer of patients to the general ward combined with an improvement in the overall quality of care.

Mechanical ventilation is one of the most important ICU treatments, and the duration of mechanical ventilation is closely related to ICU LOS. In this study, however, the number of ventilator-free days was not increased after implementing an intensivist model, probably because of the typically short duration of postoperative mechanical ventilation.

The model of a high-intensity ICU managed by an intensivist is uncommon despite its many positive impacts $[10,11,17]$. In particular, implementing an intensivist is rare in smaller hospitals and surgical ICUs $[10,18]$. Small hospitals may be keenly aware of the added costs of hiring intensivists, making them hesitant to implement an intensivist-directed model ICU. In a surgical ICU, the primary surgeons are usually reluctant to relinquish care of postoperative patients to another physician and prefer to provide the care themselves, thereby maintaining continuity of care $[10,19]$. The intensivist of this study was a surgery-based intensivist who had the advantage of understanding surgical procedures and anatomy [20]. Furthermore, the intensivist tried to improve communication and decrease conflicts with the primary surgeon, and these efforts were essential in establishing the intensivist-directed surgical ICU. Due to the shortage of intensivists in many countries, implementing an intensivist model may not be feasible in many hospitals at this time. In Korea, board certification in critical care medicine was not approved until 2008. According to a survey of the ICU system in Korea, only $17.3 \%$ of total ICUs were managed by specialized intensivist [21]. As this shortage of intensivists makes it difficult to maintain the quality of critical care, critically ill patients may be unable to receive appropriate medical treatment. The advantages of an intensivist and the high-intensity model ICU should be recognized and used to develop novel staffing paradigms in order to provide safe and appropriate care to critically ill surgical patients [17].

The present study has some limitations. First, because retrospective data were used, this study has an inherent bias. Although there was an inevitable selection bias due to the nature of a before and after intervention study, the clinical characteristics of patients were not different before and after implementation period. In this study, the APACHE II score was not significantly different between the two periods nor was the severity levels of the patients admitted in both periods. Second, there is the inevitable time difference inherent in a before and after intervention study, and the concurrent advances in medical knowledge, procedures, or drugs might contribute to better outcomes for the later patients. Furthermore, the experience levels of medical personnel likely increase over time. We cannot exclude the positive effects of these factors. However, there were no major treatment or policy changes implemented between the periods of the study, and the separation of study periods by only one year minimized the chance of the more recent patient population having access to higher levels of technology and medical therapeutics. Moreover, the clinical outcomes of the first half and latter half of each years was not different (data not shown), we may indirectly excluded the effect of the advances in medical practices.

In conclusion, we found that implementing an intensivistdirected surgical ICU was associated with improvements in ICU mortality and significant clinical outcomes. Despite several limitations, these data have significant practical and policy implications, and we believe that our results strongly support implementation of an intensivist-directed ICU in a surgical ICU.

\section{CONFLICTS OF INTEREST}

No potential conflict of interest relevant to this article was reported. 
1. Pronovost $\mathrm{P}$, Thompson DA, Holzmueller CG, Dorman T, Morlock LL. Impact of the Leapfrog Group's intensive care unit physician staffing standard. J Crit Care 2007:22:89-96.

2. Pronovost PJ, Angus DC, Dorman T, Robinson KA, Dremsizov TT, Young TL. Physician staffing patterns and clinical outcomes in critically ill patients: a systematic review. JAMA 2002;288:2151-62.

3. Carson SS, Stocking C, Podsadecki T, Christenson J, Pohlman A, MacRae S, et al. Effects of organizational change in the medical intensive care unit of a teaching hospital: a comparison of 'open' and 'closed' formats. JAMA 1996;276:322-8.

4. Ghorra S, Reinert SE, Cioffi W, Buczko G. Simms HH. Analysis of the effect of conversion from open to closed surgical intensive care unit. Ann Surg 1999:229:163-71.

5. Nathens AB, Rivara FP, MacKenzie EJ, Maier RV, Wang J, Egleston B, et al. The impact of an intensivist-model ICU on trauma-related mortality. Ann Surg 2006;244:545-54.

6. Dimick JB, Pronovost PJ, Heitmiller RF, Lipsett PA. Intensive care unit physician staffing is associated with decreased length of stay, hospital cost, and complications after esophageal resection. Crit Care Med 2001;29:753-8.

7. Kumar K, Zarychanski R, Bell DD, Manji R, Zivot J, Menkis AH, et al. Impact of 24 hour in-house intensivists on a dedicated cardiac surgery intensive care unit. Ann Thorac Surg 2009;88:1153-61.
8. Mirski MA, Chang CW, Cowan R. Impact of a neuroscience intensive care unit on neurosurgical patient outcomes and cost of care: evidence-based support for an intensivist-directed specialty ICU model of care. J Neurosurg Anesthesiol 2001;13: 83-92.

9. Pronovost PJ, Jenckes MW, Dorman T, Garrett E, Breslow MJ, Rosenfeld BA, et al. Organizational characteristics of intensive care units related to outcomes of abdominal aortic surgery. JAMA 1999; 281:1310-7.

10. Angus DC, Shorr AF, White A, Dremsizov TT, Schmitz RJ, Kelley MA, et al. Critical care delivery in the United States: distribution of services and compliance with Leapfrog recommendations. Crit Care Med 2006;34:1016-24.

11. Nathens AB, Maier RV, Jurkovich GJ, Monary D, Rivara FP, Mackenzie EJ. The delivery of critical care services in US trauma centers: is the standard being met? J Trauma 2006;60:773-83.

12. Levy MM, Rapoport J, Lemeshow S, Chalfin DB, Phillips G, Danis M. Association between critical care physician management and patient mortality in the intensive care unit. Ann Intern Med 2008;148:801-9.

13. Schoenfeld DA, Bernard GR; ARDS Network. Statistical evaluation of ventilator-free days as an efficacy measure in clinical trials of treatments for acute respiratory distress syndrome. Crit Care Med 2002;30:1772-7.
14. Gajic O, Afessa B. Physician staffing models and patient safety in the ICU. Chest 2009;135:1038-44.

15. Kahn JM, Brake H, Steinberg KP. Intensivist physician staffing and the process of care in academic medical centres. Qual Saf Health Care 2007;16:329-33.

16. Hawari FI, Al Najjar TI, Zaru L, Al Fayoumee W, Salah SH, Mukhaimar MZ. The effect of implementing high-intensity intensive care unit staffing model on outcome of critically ill oncology patients. Crit Care Med 2009:37:1967-71.

17. Ewart GW, Marcus L, Gaba MM, Bradner RH, Medina JL, Chandler EB. The critical care medicine crisis: a call for federal action: a white paper from the critical care professional societies. Chest 2004;125:1518-21.

18. Gutsche JT, Kohl BA. Who should care for intensive care unit patients? Crit Care Med 2007;35(2 Suppl):S18-23.

19. Stawicki SP, Pryor JP, Hyams ES, Gupta R, Gracias VH, Schwab CW. The surgeon and the intensivist: reaching consensus in intensive care triage. J Surg Educ 2007;64: 289-93.

20. Johnson JL, Moore EE, Aasen AO, Rogy MA, Wang JE, Alsanea $O$, et al. The role of the surgeon as intensivist: an international perspective. Curr Opin Crit Care 2006;12:357-69.

21. Korean Society of Critical Care Medicine. 1st White paper from Korean Society of Critical Care Medicine. Seoul: Korean Society of Critical Care Medicine; 2009. 